\title{
UNA HISTORIA DE LA FILOSOFÍA LATINOAMERICANA ${ }^{1}$
}

\author{
David Sobrevilla Alcázar \\ Universidad de Lima \\ trilce@terra.com.pe
}

\begin{abstract}
Resumen
Estudio crítico sobre el libro de Carlos Beorlegui descrito en la nota 1.

PALABRAS ClAVE: filosofía latinoamericana, americanismo, filosofías de la liberación.
\end{abstract}

Abstract

Review essay about the book by Carlos Berloegui described in footnote 1.

KeYwords: Latin-American philosophy, (Latin-)Americanism, philosophies of liberation.

$\overline{R A}$

Carlos Beorlegui, profesor de antropología filosófica y de historia de la filosofía española en la Universidad de Deusto, ha publicado recientemente una extensa Historia del pensamiento filosófico latinoamericano. Una búsqueda incesante de identidad. Antes había escrito dos libros dedicados a antropología filosófica y dos al pensamiento de Juan David García Bacca.

Esta obra comprende una Introducción y nueve capítulos. En aquélla, el autor indica que, pese a que ya existe un apreciable número de monografías y artículos sobre muchos filósofos y pensadores latinoamericanos, sobre diversas épocas o historias de los países iberoamericanos más potentes filosóficamente hablando, todavía no hay ninguna investigación "que se dedique, de forma amplia y pormenorizada, a presentar lo más destacable del pensamiento filosófico latinoamericano en todas sus épocas históricas y en todas sus nacionalidades" (21). Esta comprobación es una de las razones que lo animaron a emprender este trabajo y que lo ha llevado a permanecer durante varios años en El Salvador.

En dicho país se interesó por el pensamiento de Ignacio Ellacuría y, en especial, por el de Enrique Dussel, y luego por el hispanoamericano en general y por el pensamiento indígena precolombino. En su opinión, la filosofía latinoamericana es muy poco

1 Carlos Beorlegui, Historia del pensamiento filosófico latinoamericano. Una búsqueda incesante de identidad. Bilbao: Universidad de Deusto, 2004; 895 pp. 
conocida y valorada no solo en Europa sino también en la propia América Latina, cuyos "profesionales [en filosofía, D.S.] conocen mucho mejor la historia de la filosofía europea y norteamericana que la hispanoamericana" (23). Por ello ha querido componer un manual sin mayores pretensiones que la de servir de introducción a la historia de las ideas y del pensamiento filosófico hispanoamericano.

El libro se subtitula "Una búsqueda incesante de la identidad", pues el autor sostiene que este empeño impulsa en gran medida a los pensadores iberoamericanos más significativos aun desde antes de la emancipación política de España y Portugal; $\mathrm{y}$, en todo caso, a quienes conforman la tendencia americanista, que es la que ha seguido con más atención (24).

Revela que su selección da preferencia asimismo en primer lugar a los autores que están en una línea americanista; y, en segundo lugar, a los más significativos de entre ellos. Ha preferido referirse al "pensamiento filosófico latinoamericano" y no a la mera "filosofía latinoamericana", para no limitar su historia, ni el elenco de personajes que presenta en ella (25); y emplea el adjetivo "latinoamericano/a" y no otro, porque le parece el más adecuado. También confiesa utilizar un criterio amplio al extender el tratamiento del "pensamiento filosófico latinoamericano" a la Colonia e incluso a la época precolombina.

En cuanto a la periodización, ha compaginado diversos criterios: en el caso del pensamiento precolombino, la pauta ha sido la unidad histórica de cada cultura; el período colonial lo ha estudiado unitariamente; el siglo XIX lo ha dividido en dos partes: la primera comprende el pensamiento de los líderes independentistas y el romanticismo; y la segunda, el krausismo y el positivismo; y en el siglo xx ha tenido en cuenta una perspectiva generacional.

Todos estos puntos más bien metodológicos los trata Beorlegui con detalle en el primer capítulo de su libro.

El segundo capítulo está destinado a presentar sintéticamente las cosmovisiones indígenas hasta el momento de la llegada de los conquistadores europeos. Se trata de una presentación descriptiva, pero en la que también refiere la tesis de investigadores como Miguel León Portilla, quien cree haber encontrado indicios de reflexión filosófica antes de la llegada de los españoles.

El capítulo tercero estudia la filosofía durante la colonia española y portuguesa. El autor se refiere así al "descubrimiento" de América y a los problemas filosóficos que planteó, a la fundación de universidades y centros superiores de formación, a la enseñanza de la filosofía durante la época colonial y a los primeros filósofos hispanoamericanos. Sostiene -con J.D. García Bacca-que sus rasgos más significativos fueron la conciencia que tuvieron de América y de la libertad.

"El pensamiento filosófico durante la primera mitad del siglo xIX. El proceso de emancipación política y cultural" es el tema del capítulo cuarto. Muestra aquí Beorlegui cómo, con la independencia política, los intelectuales más valiosos y conscientes se orientaron sin miedo hacia la independencia cultural como meta a perseguir para lograr una segunda independencia, abominando - con contadas excepciones-de la tradición 
cultural española. Los inicios de la filosofía americana se encontrarían en el romanticismo y, más en concreto, en la reflexión pionera del argentino Juan Bautista Alberdi.

El capítulo quinto está dedicado a examinar la segunda mitad del siglo xIx y el pensamiento krausista y positivista latinoamericano. Se cierra con el tratamiento del pensamiento filosófico-político de José Martí que se sitúa por encima de ambas corrientes, y representa una síntesis teórica sobre la situación de la América hispana y una propuesta positiva en el sentido de concreta: apegada a las necesidades de Nuestra América y creadora (339-340).

"El pensamiento filosófico en los inicios del siglo xx" es estudiado en el capítulo sexto. Según el autor, el grupo generacional al que denomina de 1900 -que está integrado por Rodó y Vaz Ferreira en el Uruguay, por Justo Sierra en México, Alejandro Korn y Rivarola en la Argentina, Deustua en el Perú, Farías Brito y Graça Aranda en el Brasil y Enrique Molina en Chile- "constituye el primer punto de arranque del desmarque generalizado que se produce frente al positivismo en toda Latinoamérica" (398).

La labor cumplida por la generación de 1900 para romper con el positivismo fue proseguida, según Beorlegui, por la posterior generación de 1915, cuyo desempeño presenta en el capítulo séptimo. Esta generación habría tenido un núcleo mexicano (con autores como Caso, Vasconcelos y Reyes), otro argentino (cuya figura más destacada fue Coriolano Alberini) y uno peruano (integrado por Mariátegui, Haya de la Torre y Belaúnde) y representantes en Puerto Rico y Brasil. El autor observa que mientras Francisco Miró Quesada Cantuarias reúne a las generaciones de 1900 y 1915 en un solo grupo, el de los "patriarcas", él prefiere separarlos (479).

El mismo Miró Quesada denominaba a la generación posterior de los años 30 la de los "forjadores"; Beorlegui la estudia en el capítulo octavo de su libro. Esta generación "se centrará ya más claramente [que en superar el positivismo, D.S.] en asimilar lo más significativo de la historia de la filosofía con objeto de llegar a una normalización de la filosofía en el ámbito latinoamericano. Es el objetivo que se proponen el argentino Francisco Romero y el mexicano Samuel Ramos, tarea en la que colaboraron de modo muy significativo los exiliados españoles llegados por esos años a diversas naciones hispanoamericanas, sobre todo a México, y entre los que destacan José Gaos y Juan David García Bacca" (28-29).

El capítulo noveno está dedicado a "El grupo generacional de 1950-60". Adoptando una propuesta de F. Miró Quesada, sostiene Beorlegui que este grupo se echó a la búsqueda de una filosofía auténtica, pero que en el intento de lograrla se bifurcó (palabra que el investigador español no emplea) en dos frentes: el asuntivo, de quienes creían que todavía no era posible hacer una filosofía genuina, por lo que se limitaron a asumir la filosofía occidental; y el afirmativo, de quienes pensaban que ya se podía filosofar con originalidad a partir de la propia situación y circunstancia. La línea afirmativa habría tenido tres núcleos: el mexicano de Leopoldo Zea y el grupo "Hiperión", el peruano de A. Salazar Bondy y F. Miró Quesada Cantuarias, y el argentino-uruguayo de A. A. Roig y A. Ardao.

Los planteamientos de los principales autores de la línea afirmativa del grupo generacional de 1950-60 sirvieron de referencia y orientación a la generación siguiente, 
la de los años setenta, originadora de la filosofía de la liberación. Beorlegui le dedica el capítulo décimo, y sostiene que ha sido "la más brillante y original, junto con la anterior, de toda la historia latinoamericana" (29).

Surgida al calor de un momento histórico clave: la toma de conciencia colectiva de la situación de dependencia del continente iberoamericano, [sus integrantes, D.S.] trataron de construir un pensamiento que se autointerpreta como el momento histórico de un empeño político de liberación. Según ellos, el filósofo se convierte en alguien que escucha el ansia liberadora del pueblo y le acompaña en su empeño de llevar a la práctica tal ideal liberador. Pero, como veremos a lo largo del capítulo décimo, no todos los integrantes de esta corriente de pensamiento entenderán esta filosofía de la misma manera, advirtiéndose entre ellos diferentes sectores y escuelas dentro de la misma, en función del suelo filosófico en el que apoyan su pensamiento, el sujeto y el objeto de su filosofar, y el enfoque metodológico desde el que elaboran sus planteamientos teóricos (29).

El capítulo undécimo y último se titula “¿Más allá de la filosofía de la liberación? La postmodernidad y la postcolonialidad". Inicialmente, el autor echa una mirada panorámica a las distintas manifestaciones filosóficas actuales latinoamericanas, pero luego se concentra en "El proceso evolutivo y autocrítico al interior de la Filosofía de la Liberación". Aquí trata de la propuesta de filosofía intercultural de Raúl FornetBetancourt. Serían, sin embargo, los enfoques posteriores de la filosofía de la postmodernidad y de la postcolonialidad, que desde "las últimas décadas del siglo xx, pretenden disputar a la filosofía de la liberación su carácter hegemónico dentro del panorama filosófico latinoamericano" y los "últimos paradigmas culturales con peso desde los que se pretende en la actualidad interpretar la situación sociocultural de la América Latina" (29).

La sección última del capítulo undécimo se titula "La identidad de la razón latinoamericana", y en ella explica Beorlegui algunas conclusiones generales que recogen el significado de la trayectoria histórica del pensamiento filosófico latinoamericano. Ellas son: 1) Recuerda que ha emprendido su Historia con un criterio amplio, tratando así del "pensamiento filosófico latinoamericano" y no solo de la "filosofía latinoamericana". 2) "Esta problemática inicial ha supuesto que lo específico del pensamiento o de la filosofía latinoamericana haya sido en gran medida a lo largo de su historia la búsqueda de su identidad" (892). 3) "Una constatación importante de este proceso histórico posterior a la emancipación política es la periódica reflexión sobre si cabe una filosofía auténticamente latinoamericana, y cuáles podrían ser los rasgos específicos de la misma" ( 882). A veces se ha supuesto que exista un tronco esencial del filosofar, el greco-occidental, al cual se tienen que injertar los demás tipos de filosofar; pero los resultados de la reflexión latinoamericana sobre la específica identidad del pensamiento latinoamericano muestran el error radical de este planteamiento. Así como Aristóteles sostuvo que el ser se dice de numerosas maneras, sugiere Beorlegui que también la razón; y que una de sus variedades es la razón latinoamericana. 4) “... una de las mejores aportaciones de la razón latinoamericana es la mostración de que las aportaciones que una cultura puede hacer a la universalidad pasan por una 
profundización en lo local y en lo específico de su propia cultura" (883). 5) Las características sobresalientes del filosofar latinoamericano son las siguientes: a) el filosofar desde la propia circunstancia, b) la referencia práctica del pensamiento, c) la orientación humanística, d) la fuerte tendencia ética, e) el horizonte universal de sus planteamientos, f) "Como balance final, creemos que es de justicia reconocer que el pensamiento filosófico latinoamericano se encuentra en un excelente momento de madurez, en el que se habría producido la pretendida normalización que ansiaban los intelectuales de la generación de los Fundadores, en terminología de Francisco Miró Quesada" (884).

Los méritos de este libro de Carlos Beorlegui son muchos. Uno de los más saltantes es que con él ha proporcionado la primera historia del pensamiento filosófico latinoamericano escrita en España. En efecto, ya existían recuentos parecidos hechos en otros países europeos: en Italia (el libro de Sergio Sarti Panorama della filosofia hispanoamericana contemporánea. Milán: Cisalpino-Golliardica, 1976); Francia (la obra de Alain Guy Panorama de la Philosophie Ibéro-Américaine. Du XVIe Siècle à nos jours. Ginebra: Patiño, 1989) y Alemania (el estudio de Heinz Krumpel Philosophie in Lateinamerika. Grundzüge ihrer Entwicklung. Berlín: Akademie-Verlag, 1992)². No existe nada semejante en Estados Unidos o en Gran Bretaña - que sepamos- sino solo repertorios de textos como el editado por Jorge J. E. Gracia Latin-American Philosophy in the Twentieth Century. Man, Values and the Search for Philosophical Identity (Buffalo: Prometheus Books, 1986). Pues bien, aunque pueda resultar chocante -dada la cercanía en que se supone que está la cultura española de la latinoamericana- tampoco existía ninguna historia del pensamiento filosófico latinoamericano hecha en España. Son precisamente libros como éste de Beorlegui -o la Enciclopedia Iberoamericana de Filosofía, empezada a publicar en 1992 por el Instituto de Filosofía del Consejo Superior de Investigaciones Científicas de Madrid junto con el Instituto de Investigaciones Filosóficas de la Universidad Autónoma de México y el Centro de Investigaciones Filosóficas de Buenos Aires-, los que fundan la vinculación real entre el filosofar español y el latinoamericano. No es poca cosa.

Por otro lado, la Historia de Beorlegui procesa una enorme cantidad de material y lo hace de una manera bastante didáctica -aunque la elección de sus formas expresivas no sea siempre la mejor. A ello se agrega que algunas de sus exposiciones están bien hechas y versan sobre autores no muy trabajados - pienso por ejemplo en las presentaciones de las ideas de Enrique Dussel o de Juan Carlos Scannone. Sin duda, con todo ello contribuye el autor a hacer más conocida y mejor valorada la filosofía

2 Es realmente sorprendente que Beorlegui ignore en las bibliografías que cierran cada capítulo de su obra estos libros y que, a la vez, afirme que no hay ninguna investigación de conjunto que proporcione un panorama de lo más destacable del pensamiento filosófico latinoamericano, que es justamente lo que estas obras ofrecen. En América Latina existen por cierto otros recuentos más, como por ejemplo: Abelardo Villegas, Panorama de la filosofía iberoamericana actual (Buenos Aires: Eudeba, 1963), que tampoco cita el autor. Él solo menciona el recuento de Leopoldo Zea, El pensamiento Latinoamericano (Barcelona: Ariel, ${ }^{3} 1976$ ), el pequeño libro de Alain Guy La filosofía en América Latina (Madrid: Acento, 1997) y la obra colectiva Filosofía en América Latina (La Habana: Félix Varela, 1998). 
latinoamericana, pues tiene toda la razón cuando menciona que es ignorada y minusvalorada no solo en Europa y los Estados Unidos, sino también en la misma América Latina.

Veamos ahora los problemas del libro. Los primeros son de índole metodológica. Ante todo: su enorme vaguedad en el empleo de algunas palabras y expresiones clave, como por ejemplo, "pensamiento filosófico latinoamericano", "identidad" o "identidad cultural", o "razón" y "razón latinoamericana", que no aclara en forma satisfactoria. Como Beorlegui no llega a explicar en forma precisa lo que entiende por "pensamiento filosófico" a diferencia de una genuina "filosofía", pese a su consideración introductoria de la página 38, puede incluir en este libro el pensamiento de los nahuas, mayas e incas, pero a la vez criticar a León Portilla por haber hablado de una "filosofía náhuatl" -aunque sin discutir sus razones (93). Por otro lado, pasa revista a algunas tesis sobre la identidad latinoamericana (pp. 46-49), mas sin arribar a ninguna conclusión al respecto. Tampoco explica cómo así la razón se dice de numerosas maneras, una de las cuales es la variedad de la "razón latinoamericana". Que afirme que ésta filosofa a partir de su propia circunstancia pero que a la vez mira siempre al entorno universal (883-884), no es muy iluminador: ya Montesquieu sostenía que la razón tiene en cuenta las relaciones necesarias entre las cosas, pero a la vez sus circunstancias -lo que este autor denominaba su "espíritu"-, por lo que estos rasgos no parecen ser específicos de la presunta razón latinoamericana.

Por otra parte, al autor le falta sin duda familiaridad con el pensamiento filosófico latinoamericano - que es probable que únicamente hubiera podido tratar con solvencia en España un gran conocedor y scholar como José Luis Abellán. Me referiré casi solo a algunas ausencias y exabruptos de Beorlegui en su presentación de la filosofía peruana: ignora la importancia de la ponencia de Francisco García Calderón "Las corrientes filosóficas en la América Latina" (1908), un texto esencial desde una perspectiva americanista; no hace ninguna mención a Mariano Iberico, Pedro Zulen, José Russo Delgado y Víctor Li Carrillo que son cuatro importantes filósofos peruanos - la mayoría de los cuales son tratados o mencionados en las obras sobre filosofía latinoamericana de Sarti, Guy o Krumpel e igualmente en el Diccionario de Filosofía de José Ferrater Mora; en la página 582 nos informa que uno de los centros de la filosofía analítica en el Perú es la Universidad Peruana Cayetano Heredia y su Instituto de Investigaciones Filosóficas y que allí se está formando una nueva generación de pensadores, sin percatarse de que dicha Universidad cerró su especialidad de filosofía y su Instituto de Investigaciones Filosóficas hace un cuarto de siglo (en 1982!). Podríamos proseguir esta lista. Por cierto, los gazapos también se producen en el caso de las filosofías o filósofos de otros países; así, por ejemplo, el filósofo argentino Rodolfo Agoglia resulta ser para Beorlegui un "escritor ecuatoriano" (46); y al pensador uruguayo Juan Llambías de Azevedo lo ubica "en el entorno brasileño" (563).

Una tercera dificultad metodológica es el desconocimiento por parte de Beorlegui, o en todo caso su falta de utilización, de la enorme cantidad de bibliografía existente sobre las filosofías nacionales, sobre algunas corrientes o figuras. La hay en Argentina, México, Perú, Colombia, Chile y en otros países más, pero el autor simplemente la ignora. 
Una cuarta dificultad metodológica es que el autor incluya en esta Historia del pensamiento filosófico "latinoamericano" al Brasil. Probablemente haya razones para hacerlo, pero esta opción sobreexige el nivel actual de conocimiento de los historiadores del pensamiento de Nuestra América y, sin duda, el del historiador español de las ideas.

Y, finalmente, hay una cierta predilección en Beorlegui por subrayar la influencia de la filosofía española en América Latina, en especial la de Ortega y Gasset, José Gaos y Juan David García Bacca. Pensamos que tiene razón en cuanto a la audiencia que logró Ortega, aunque también habría que mencionar que despertó enormes resistencias -y no solo en la Argentina. Sobre la influencia de Gaos y de García Bacca opinamos que fue en especial como traductores y, en el caso del primero, como suscitador de vocaciones.

Mas, la mayor dificultad de esta Historia se halla en otro lado: en la visión sesgada que ofrece del pensamiento filosófico latinoamericano y de su desarrollo. Es plenamente legítimo que el autor haya privilegiado un determinado punto de vista para seleccionar su material y él lo expone con toda honestidad: ha seguido con una mayor atención la tendencia americanista dentro del pensamiento filosófico latinoamericano, dejando en segundo término a los autores y a las obras "que no se han escrito dentro de este horizonte teórico" (24). Lo criticable es que esta perspectiva provoque una distorsión tan severa que hace que esta Historia del pensamiento filosófico latinoamericano se convierta en verdad en una Historia del pensamiento filosófico liberacionista latinoamericano o, en el mejor de los casos, en una Historia del pensamiento filosófico latinoamericano desde la perspectiva de la filosofía de la liberación.

La distorsión se produce tanto en relación con el pasado como con el presente del pensamiento filosófico latinoamericano. En relación con el pasado, porque Beorlegui solo lleva a cabo un recuento pasivo del mismo, ya que en su opinión por entonces no existía la línea americanista. Para él, la filosofía americana auténtica recién surgió con Juan Bautista Alberdi (28): todo lo anterior -es decir, el pensamiento precolombino y el colonial-solo fue un preámbulo sin mayor significación. De allí que el autor no preste casi ninguna importancia a los hallazgos de la lógica mexicana o peruana colonial, como sí han hecho otros investigadores ${ }^{3}$; o a otros tópicos de pareja importancia en el pensamiento en los Virreinatos de la Nueva España y del Perú 4 .

3 Como Walter Redmond o Mauricio Beauchot. V. las investigaciones de Redmond y Beauchot, La lógica mexicana en el siglo de oro (México: UNAM, 1985) y de Redmond, La lógica en el Virreinato del Perú (Lima: FCE/PUC, 1998).

4 En una reseña que entendemos que todavía está inédita, María Luisa Rivara de Tuesta afirma que por ejemplo Beorlegui no ha tenido para nada en cuenta "la importancia del pensamiento humanista cristiano que logró, a través de un largo debate, el reconocimiento de la naturaleza racional y humana del natural americano, e, igualmente, la aceptación de las tres culturas existentes en América y que, por lo tanto, los naturales no podían ser vendidos como esclavos". 
Pero tampoco estudia el período postcolonial en su real importancia: piénsese en los grandes vacíos que muestra esta Historia en cuanto al pensamiento cristiano, marxista, a la filosofía de la ciencia, etc.

Mas, la distorsión mayor se produce en torno al pensamiento filosófico latinoamericano actual -entendiendo por tal, sin mucha precisión al que se despliega a partir de mediados del siglo xx hasta hoy. En efecto, revísense los capítulos noveno (que trata del grupo generacional 50-60), décimo (que se ocupa de la generación de los años setenta) y undécimo (que presenta la filosofía posterior), y se comprobará que la técnica de la presentación es la misma: una mirada panorámica apresurada sobre la filosofía no americanista, y luego el resto de los respectivos capítulos está dedicado a tratar pormenorizadamente las manifestaciones filosóficas americanistas: la filosofía de lo americano, la de la liberación y la postliberacionista. La gran figura de la filosofía latinoamericana resulta ser así Enrique Dussel, con quien se produce una apoteosis filosófica: primero con su filosofía de la liberación y hoy en día con su heredera, la filosofía postcolonial.

La distorsión que este procedimiento genera es enorme. En un libro de 895 páginas sobre el pensamiento filosófico latinoamericano, cualquier lector tiene el derecho de esperar conocer a sus mayores filósofos y de encontrar una presentación equilibrada de sus principales corrientes y de sus figuras nacionales más importantes; pero ello no ocurre en el caso de la Historia de Carlos Beorlegui. Grandes figuras de la filosofía latinoamericana, como Mario Bunge, Héctor Neri Castañeda, Risieri Frondizi, Ernesto Laclau, Newton da Costa apenas son tratadas. Una de las manifestaciones más importantes de la filosofía latinoamericana ha sido en las décadas recientes la filosofía analítica argentina del derecho, integrada por notables jusfilósofos como Ambrosio L. Gioja, Genaro Carrió, Roberto Vernengo, Carlos Alchourrón, Eugenio Bulygin y Carlos S. Nino; pero de ello no se entera el lector en esta Historia. Otra orientación importante en América Latina ha sido la de los cultores de la filosofía de la ciencia, con autores tan prestigiosos como Adolfo García de la Sienra, Carlos Ulises Moulines, León Olivé, Ana Rosa Pérez Ransanz, Alberto Cordero, Miguel Espinoza, Sergio Ramírez, entre otros más; pero poca mención hay a ellos en esta obra. Las ausencias de los grandes representantes de las distintas filosofías nacionales o la poca atención que se les concede son todavía más llamativas: probablemente los dos filósofos recientes más importantes en Colombia sean Nicolás Gómez Dávila y Danilo Cruz Vélez, pero solo el último es mencionado fugazmente en esta Historia. En Chile, grandes figuras últimas, como Roberto Torretti, Carla Cordua, Alfonso Gómez Lobo, Humberto Giannini o Pablo Oyarzún, son casi ignoradas. Para hacer una analogía: ¿cómo se consideraría una Historia de la Filosofía Europea que solo se centrara en la tradición marxista y que afirmara que la gran filosofía del siglo xx ha sido la de la Escuela de Frankfurt, dando un lugar insignificante a la fenomenología o a la filosofía analítica y a figuras como Husserl o Heidegger, Russell, Carnap o Wittgenstein?

La distorsión se produce también de otra manera. Algunos filósofos latinoamericanos sí son tratados con alguna amplitud, pero solo en cuanto han tenido que ver con la línea americanista; sus otros logros son sencillamente silenciados. Así se entera 
el lector de la obra de Francisco Miró Quesada Cantuarias, pero solo en tanto se aproximó a la filosofía de la liberación (642-643); poca mención hay de que fue uno de los introductores de la lógica formal en América Latina, uno de los pioneros a nivel mundial de la aplicación de la lógica formal al derecho, que ha tratado de desarrollar una novedosa teoría de la razón. También se informa sobre el aporte de Augusto Salazar Bondy como uno de los antecesores de la filosofía de la liberación (624 ss.); pero no se dice que escribió la mejor historia de las ideas en el Perú contemporáneo, o de que tiene un gran planteamiento analítico sobre ética. De manera semejante, el lector toma conocimiento de que Luis Villoro polemizó con Leopoldo Zea (597 ss.) y de sus planteamientos cuando pertenecía al grupo "Hiperión" (610-612); pero apenas se menciona que ha escrito dos libros notables sobre filosofía del conocimiento y ética y política (Creer, saber, conocer y El poder y el valor). Es decir que las ideas de Miró Quesada, Salazar Bondy y Villoro solo son importantes para este libro cuando estos autores estuvieron alguna vez próximos a la filosofía de la liberación; the rest is silence.

Mas la distorsión tiene lugar también de otra manera: al destacar desmesuradamente Beorlegui a figuras que no tienen la figuración que les atribuye. Liberacionistas como Kusch y Scannone, Dussel o Cerutti, Roig o Hinkelammert son bastante conocidos y reconocidos por sus obras y amplia trayectoria. Pero, sin duda, la significación que esta Historia concede a Raúl Fornet-Betancourt y a su filosofía intercultural-que es en verdad un desarrollo de la filosofía intercultural europea ${ }^{5}$ - es excesiva: Beorlegui le concede 14 páginas frente a las pocas líneas que destina a Mario Bunge. O dedica también largas páginas al filósofo postcolonialista colombiano Santiago Castro Gómez (841-877), en tanto que apenas se ocupa de otros filósofos colombianos que no tienen una orientación americanista, como Rubén Sierra Mejía, Carlos Gutiérrez, Juan José Botero, Luis Eduardo Hoyos o Rodolfo Arango, pero que en su país son bastante más conocidos.

Lo anterior muestra que ésta es una Historia excesivamente "ideológica": que deforma la realidad y el desarrollo del pensamiento filosófico latinoamericano debido a su enfoque sesgadamente americanista. Preguntémonos entonces si ésta es -cuando menos- una buena Historia del pensamiento filosófico liberacionista latinoamericano. Lamentablemente, tenemos la impresión de que no es así. Por una parte, Beorlegui no ha conducido una investigación propia que rastree en América Latina el surgimiento y evolución de la filosofía liberacionista, sino que simplemente se limita a orientarse por las obras ya existentes sobre el particular, como las de Horacio Cerutti Guldberg y Enrique Dussel ${ }^{6}$. Si hubiera hecho una investigación autónoma, hubiera encontrado

5 La filosofía intercultural surgió hacia 1988 en Austria y Alemania de los esfuerzos, sobre todo, de Franz E. Wimmer y Ram Adhar Mall. V. los libros de Wimmer, Vier Fragen zur Philosophie in Afrika, Asien und Lateinamerika (1988), R.A. Mall/H. Hülsmann, Die drei Geburtsorte der Philosophie, China, Indien, Europa (1983) y R. A. Mall, Philosophie im Vergleich der Kulturen (1995).

6 V. Horacio Cerutti Guldberg, Filosofía de la liberación latinoamericana (México: FCE, 1983; ${ }^{2} 1992 ;{ }^{32006)}$ y Enrique Dussel, Historia de la filosofía y filosofía de la liberación (México: Nuestra América, 1994). 
que el panorama de la filosofía liberacionista es bastante más complejo de cómo lo presenta; por ejemplo, que en el Perú hay una propuesta de filosofía inculturada independiente de la de Scannone, procedente de Noé Zevallos en su libro Apuntes para una antropología liberadora (Bogotá: CLAR, 1977). Por otra parte, otra forma de distorsión se hace aquí presente. Según Horacio Cerutti Guldberg, en la filosofía de la liberación hay cuatro corrientes, a las que denomina "ontologista", "analéctica", "historicista" y "problematizadora". De todas ellas, la privilegiada por Beorlegui es la "analéctica" de Scannone y Dussel, y de éstos, su héroe es sin duda Dussel. Con lo cual resultan minimizadas las propuestas de A. A. Roig o de Leopoldo Zea en su fase liberacionista. Por último, la presentación que hace Beorlegui del desarrollo de la filosofía de la liberación en el capítulo 11, después de mostrar su génesis en los años 70 en el capítulo 10, es enormemente problemática. El autor manifiesta que la filosofía de la liberación se halla en un proceso de permanente evolución (814-817). Luego presenta la propuesta de la filosofía intercultural de Raúl Fornet-Betancourt (817-831) y a continuación, la filosofía postmoderna latinoamericana (831-857) y el nuevo paradigma de la "postcolonialidad" (857-877). Serían estas dos últimas filosofías las que "pretenden disputar a la filosofía de la liberación su carácter hegemónico dentro del proceso filosófico latinoamericano". Termina manifestando que actualmente hay algunas comunidades entre la filosofía de la liberación y la filosofía postmoderna latinoamericana y la de la postcolonialidad (878-879), pero un número todavía mayor de diferencias significativas. Pues bien, tenemos la impresión de que esta presentación diverge mucho de la realidad actual de la filosofía de la liberación: ante todo, porque ésta ha dejado de existir como una unidad hace mucho tiempo, como lo constataba ya en 1988/89 Horacio Cerutti Guldberg 7 ; y lo corrobora el propio Beorlegui cuando reconoce los cambios profundos que se han operado en el pensamiento de los principales filósofos liberacionistas: J. C. Scannone, E. Dussel, L. Zea, A. A. Roig, F. Hinkelammert e I. Ellacuría (Historia: 815). Por otra parte, su exposición es curiosa: ¿por qué presentar a la filosofía intercultural de Raúl Fornet-Betancourt como si fuera un simple desarrollo de la filosofía de la liberación, cuando de la propia descripción del desarrollo del filósofo cubano que hace Beorlegui se observa que no tuvo una fase liberacionista y que, en todo caso, se ha desmarcado de la filosofía de la liberación? Y por último, ¿quiénes son los filósofos liberacionistas que hoy reivindican la hegemonía de la filosofía de la liberación frente al desafío de la filosofía postmoderna latinoamericana y, sobre todo, de la filosofía postcolonialista, si algunos de los filósofos liberacionistas latinoamericanos de los años 70 ya han muerto (Kusch, Ellacuría, Zea), si otros han desarrollado filosofías que seguramente son evoluciones plenamente legítimas de la filosofía de la liberación, pero con rasgos bastante diferentes (Scannone, que ha propuesto una filosofía inculturada, y Dussel, que defiende la filosofía postcolonial y

7 Escribía en su artículo "Situación y perspectivas de la filosofía para la liberación latinoamericana" (ahora en: H. Cerutti Guldberg, Filosofías para la liberación ¿liberación del filosofar? México: UAEM, 1997: [Actualmente] "Es prácticamente imposible hablar de una filosofía de la liberación y, más bien, habría que hablar de filosofías (múltiples y variadas en sus especies) para (que colaboran con el proceso de) la liberación latinoamericana" (190) 
una versión de la ética distinta a la que inicialmente había desarrollado ${ }^{8}$ ), y, finalmente, cuando unos terceros, como A. A. Roig y H. Cerutti, han desarrollado su labor sobre todo en el campo de la historia de las ideas?

Probablemente este capítulo 11 sea el más frustrante del libro, pero no solo por la presentación anterior, que expone la situación presente del pensamiento latinoamericano entre la filosofía de la liberación, la postmoderna y la postcolonial sin apreciar en su justo valor toda la multiplicidad de manifestaciones filosóficas latinoamericanas actuales, sino por sus conclusiones. Sus tesis más fuertes son que la especificidad del pensamiento filosófico latinoamericano reposa en la búsqueda de su identidad, la que se encontraría en el descubrimiento de la peculiaridad de la razón latinoamericana. $L a$ identidad buscada sería la de la razón latinoamericana. Pero, ¿cómo entender estas tesis si Beorlegui no ha explicado - como vimos-qué es lo que entiende por identidad latinoamericana, ni tampoco cómo así la razón se dice de diversas maneras? Es cierto, en algunas ocasiones se ha hablado de que América Latina posee una razón o racionalidad alternativa a la occidental, pero que sepamos, el único proyecto en parte realizado de una nueva teoría de la razón en América Latina es el de Francisco Miró Quesada Cantuarias. Por lo demás, en este proyecto - que Beorlegui no toma en cuenta, como manifestamos- no se trataba de plantear una razón alternativa a la occidental, sino de formular en efecto una nueva teoría de la razón que supere los problemas que el surgimiento de las nuevas lógicas, la nueva física, el historicismo etc., plantean a la teoría clásica de la razón. Hasta Enrique Dussel afirma enfáticamente (como recuerda el propio Beorlegui en su Historia (pp. 878-879) que la crítica de la "razón moderna" que se halla en su filosofía de la liberación no debe ser confundida con una crítica de la razón como tal: se trataría de formular una teoría de la racionalidad que sea más universal-agrega Dussel que esta teoría de la razón debe ser a la vez diferencial ("la razón ejercida por los movimientos feministas, ecologistas, culturales, étnicos, de la clase obrera, de las naciones periféricas, etc.") (Hacia una filosofía política crítica. Bilbao: desclée, 2001: 449-450), pero nunca ha aclarado cómo se integra esta universalidad de la nueva razón en la diferencia de sus variantes.

Por todo lo anterior, hay que destacar los indudables méritos de esta Historia del pensamiento filosófico latinoamericano de Carlos Beorlegui, pero, a la vez, hay que prevenir frente al carácter sesgado de su presentación y poner en cuestión sus conclusiones.

8 Ética de la liberación en la edad de la globalización y de la exclusión. Madrid: Trotta/ UNAM/UAM, 1998: 14 